\title{
$C^{m}$ SOLUTIONS OF SYSTEMS OF FINITE DIFFERENCE EQUATIONS
}

\author{
XINHE LIU, XIULI ZHAO, and JIANMIN MA
}

\author{
Received 25 February 2002
}

\begin{abstract}
Let $\mathbb{R}$ be the real number axis. Suppose that $G, H$ are $C^{m}$ maps from $\mathbb{R}^{2 n+3}$ to $\mathbb{R}$. In this note, we discuss the system of finite difference equations $G(x, f(x)$, $f(x+1), \ldots, f(x+n), g(x), g(x+1), \ldots, g(x+n))=0$ and $H(x, g(x), g(x+1), \ldots$, $g(x+n), f(x), f(x+1), \ldots, f(x+n))=0$ for all $x \in \mathbb{R}$, and give some relatively weak conditions for the above system of equations to have unique $C^{m}$ solutions $(m \geq 0)$.
\end{abstract}

2000 Mathematics Subject Classification: 39A20, 47B38.

1. Introduction. In $[4,5,6]$, the iterative functional equations $f^{2}(z)(=$ $f(f(z)))=a z^{2}+b z+c$ and $\sum_{k=0}^{n} c_{k} f^{k}=0$ were considered, respectively. Zhang $[7,8]$ showed the existence and uniqueness of $C^{0}, C^{1}$ solutions of the equation $F(x)-\sum_{k=1}^{n} \lambda_{k} f^{k}(x)=0$. In [3], the authors studied more general iterative functional equation $G\left(x, f(x), \ldots, f^{n}(x)\right)=0$ and showed the existence, uniqueness, and stability of $C^{m}$ solutions $(m \geq 0)$ of the equation. The $C^{m}$ solutions $(m \geq 0)$ of the equation $\sum_{i=0}^{n} c_{i} f(x+i)=F(x)$ were discussed in [2]. In this note, we discuss the following system of finite difference equations:

$$
\begin{aligned}
& G(x, f(x), f(x+1), \ldots, f(x+n), g(x), g(x+1), \ldots, g(x+n))=0 \\
& H(x, g(x), g(x+1), \ldots, g(x+n), f(x), f(x+1), \ldots, f(x+n))=0
\end{aligned}
$$

for all $x \in \mathbb{R}$, where $G, H \in C^{m}\left(\mathbb{R}^{2 n+3}, \mathbb{R}\right)$ are given functions and $f, g \in C^{m}(\mathbb{R}, \mathbb{R})$ are unknown functions to be solved. Using the method of approximating fixed points by a small shift of maps, we give some relatively weak conditions for the above system of equations to have unique $C^{m}$ solutions for any integer $m \geq 0$.

Denote by $\mathbb{Z}_{+}$the set of all nonnegative integers. For $m \in \mathbb{Z}_{+}$and $k \in \mathbb{N}$, write $\mathbb{Z}_{m}=\{0,1, \ldots, m\}$ and $\mathbb{N}_{k}=\{1, \ldots, k\}$. For $f, g \in C^{0}(\mathbb{R}, \mathbb{R})$ and $r, s \in \mathbb{R}$, define the map $r f+s g: \mathbb{R} \rightarrow \mathbb{R}$ by $(r f+s g)(x)=r f(x)+s g(x)$ (for any $x \in \mathbb{R}$ ). Then, under this operation, $C^{0}(\mathbb{R}, \mathbb{R})$ is a linear space.

Let $m \geq k>0$. For $g \in C^{m}(\mathbb{R}, \mathbb{R})$, denote by $g^{(k)}$ the $k$ th derivative of $g$. Then $\boldsymbol{g}^{(k)} \in C^{m-k}(\mathbb{R}, \mathbb{R})$. Usually, $\boldsymbol{g}^{(1)}$ and $\boldsymbol{g}^{(2)}$ are written as $\boldsymbol{g}^{\prime}$ and $\boldsymbol{g}^{\prime \prime}$. In addition, for any $g \in C^{0}(\mathbb{R}, \mathbb{R})$, we put $g^{(0)}=g$ and call $g^{(0)}$ the 0 th derivative of $g$. 
Now we introduce some symbols which are defined as in [3]. For any two points $x \neq y$ in $\mathbb{R},(g(x)-g(y)) /(x-y)$ is called a difference quotient of $g$. Let

$$
\Lambda_{g}=\Lambda(g)=\{(g(x)-g(y)) /(x-y): x, y \in \mathbb{R}, x \neq y\}
$$

The set $\Lambda_{g}$ is called the set of difference quotients of $g$. If $\Lambda_{\mathfrak{g}} \subset[0, \infty)$, then $g$ is increasing; and if $\Lambda_{g} \subset(0, \infty)$, then $g$ is strictly increasing. For $g \in C^{1}(\mathbb{R}, \mathbb{R})$, it is easy to verify that

$$
\Lambda_{g} \subset g^{\prime}(\mathbb{R}) \subset \bar{\Lambda}_{g}
$$

where $g^{\prime}(\mathbb{R})=\left\{g^{\prime}(x): x \in \mathbb{R}\right\}$ and $\bar{\Lambda}_{g}$ is the closure of $\Lambda_{g}$ in $\mathbb{R}$. Write

$$
\lambda_{g}=\lambda(g)=\sup \left\{|t|: t \in \Lambda_{g}\right\}
$$

If $\lambda_{g}<\infty$, that is, $\Lambda_{g}$ is bounded, then $g$ is said to be Lipschitz continuous and $\lambda_{g}$ is called the (smallest) Lipschitz constant of $g$.

Let $m \geq j \geq 0$ be integers and let $r \geq 0$ be a real number. Suppose that $K, K_{0}$, $K_{1}, \ldots, K_{j}$ are all connected closed subsets of $\mathbb{R}$. Write

$$
\begin{gathered}
C^{m}\left(\mathbb{R}, K ; K_{0}, K_{1}, \ldots, K_{j}\right)=\left\{f \in C^{m}(\mathbb{R}, K): \Lambda\left(f^{(i)}\right) \subset K_{i}, \text { for } i=0,1, \ldots, j\right\}, \\
C^{m}(\mathbb{R}, K ; r)=\left\{f \in C^{m}(\mathbb{R}, K):|f(0)| \leq r\right\}, \\
C^{m}\left(\mathbb{R}, K ; r, K_{0}, K_{1}, \ldots, K_{j}\right)=C^{m}(\mathbb{R}, K ; r) \cap C^{m}\left(\mathbb{R}, K ; K_{0}, K_{1}, \ldots, K_{j}\right), \\
L C^{m}(\mathbb{R}, K)=\left\{f \in C^{m}(\mathbb{R}, K): \Lambda\left(f^{(i)}\right) \text { is always bounded for each } i \in \mathbb{Z}_{m}\right\} .
\end{gathered}
$$

Let $n \geq 1$. For any $G \in C^{0}\left(\mathbb{R}^{2 n+3}, \mathbb{R}\right)$ and any $i \in \mathbb{Z}_{2 n+2}$, put

$$
\begin{gathered}
\Lambda_{i G}=\Lambda_{i}(G)=\left\{\frac{G\left(y_{0}, \ldots, y_{i}, \ldots, y_{2 n+2}\right)-G\left(y_{0}, \ldots, y_{i-1}, w_{i}, y_{i+1}, \ldots, y_{2 n+2}\right)}{y_{i}-w_{i}},\right. \\
\left.\left(y_{0}, \ldots, y_{i}, \ldots, y_{2 n+2}\right) \in \mathbb{R}^{2 n+3}, w_{i} \in \mathbb{R}-\left\{y_{i}\right\}\right\}, \\
\lambda_{i G}=\sup \left\{|t|: t \in \Lambda_{i G}\right\}, \\
\lambda_{G}=\lambda_{G}^{(0)}=\max \left\{\lambda_{i G}: i=0,1, \ldots, 2 n+2\right\} .
\end{gathered}
$$

If $\lambda_{G}<\infty$, that is, each $\Lambda_{i G}$ is bounded, then $G$ is said to be Lipschitz continuous. Let the 0th-order partial derivative $G^{(0)}$ of $G$ be $G$ itself. For $G \in$ $C^{m}\left(\mathbb{R}^{2 n+3}, \mathbb{R}\right), k \in \mathbb{N}_{m}(m \geq 1)$, and $\left(i_{1}, i_{2}, \ldots, i_{k}\right) \in \mathbb{Z}_{2 n+2}^{k}$, denote by $G_{i_{1} i_{2} \cdots i_{k}}^{(k)}$ 
a $k$ th-order partial derivative of $G$, the definition of which is

$$
G_{i_{1} i_{2} \cdots i_{k}}^{(k)}\left(y_{0}, y_{1}, \ldots, y_{2 n+2}\right)=\frac{\partial^{k} G\left(y_{0}, y_{1}, \ldots, y_{2 n+2}\right)}{\partial y_{i_{1}} \partial y_{i_{2}} \cdots \partial y_{i_{k}}}
$$

for any $\left(y_{0}, y_{1}, \ldots, y_{2 n+2}\right) \in \mathbb{R}^{2 n+3}$. Obviously, $G_{i_{1} i_{2} \cdots i_{k}}^{(k)} \in C^{m-k}\left(\mathbb{R}^{2 n+3}, \mathbb{R}\right)$. In addition, we also write $G_{i_{1}}^{\prime}$ for $G_{i_{1}}^{(1)}$ and $G_{i_{1} i_{2}}^{\prime \prime}$ for $G_{i_{1} i_{2}}^{(2)}$. Let

$$
\lambda_{G}^{(k)}=\max \left\{\lambda_{H}: H \text { is a } k \text { th-order partial derivative of } G\right\} .
$$

Let $K_{0}, K_{1}, \ldots, K_{2 n+2}$ be all connected closed subsets of $\mathbb{R}$ and $m \geq 0$. Write

$$
\begin{gathered}
C^{m}\left(\mathbb{R}^{2 n+3}, \mathbb{R} ; K_{0}, K_{1}, \ldots, K_{2 n+2}\right)=\left\{G \in C^{m}\left(\mathbb{R}^{2 n+3}, \mathbb{R}\right): \Lambda_{i G} \subset K_{i}, i \in \mathbb{Z}_{2 n+2}\right\}, \\
L C^{m}\left(\mathbb{R}^{2 n+3}, \mathbb{R}\right)=\left\{G \in C^{m}\left(\mathbb{R}^{2 n+3}, \mathbb{R}\right): \lambda_{G}^{(k)}<\infty \text { for each } k \in \mathbb{Z}_{m}\right\} .
\end{gathered}
$$

If $G \in C^{1}\left(\mathbb{R}^{2 n+3}, \mathbb{R}\right)$, then analogous to (1.3), we have

$$
\Lambda_{i G} \subset G_{i}^{\prime}\left(\mathbb{R}^{2 n+3}\right) \subset \bar{\Lambda}_{i G}
$$

For convenience, we write

$$
V_{f g}(x)=(x, f(x), f(x+1), \ldots, f(x+n), g(x), g(x+1), \ldots, g(x+n))
$$

for all $f, g \in C^{0}(\mathbb{R}, \mathbb{R})$ and all $x \in \mathbb{R}$.

Let $m \geq 0$ and $G, H \in C^{m}\left(\mathbb{R}^{2 n+3}, \mathbb{R}\right)$. For real number $\delta \neq 0$, define $\Psi_{\delta G H}$ : $C^{m}(\mathbb{R}, \mathbb{R}) \times C^{m}(\mathbb{R}, \mathbb{R}) \rightarrow C^{m}(\mathbb{R}, \mathbb{R}) \times C^{m}(\mathbb{R}, \mathbb{R})$ by

$$
\Psi_{\delta G H}(f, g)=\left(\Psi_{\delta G}(f, g), \Psi_{\delta H}(f, g)\right)
$$

for all $(f, g) \in C^{m}(\mathbb{R}, \mathbb{R}) \times C^{m}(\mathbb{R}, \mathbb{R})$, where

$$
\begin{gathered}
\Psi_{\delta G}(f, g)(x)=f(x)+\delta G\left(V_{f g}(x)\right), \\
\Psi_{\delta H}(f, g)(x)=g(x)+\delta H\left(V_{g f}(x)\right),
\end{gathered}
$$

for all $x \in \mathbb{R}$. It is easy to see that $(f, g)$ is a fixed point of the map $\Psi_{\delta G H}$ if and only if $(f, g)$ is a $C^{m}$ solution of (1.1). Thus, the problem of solutions of (1.1) can be translated into that of fixed points of $\Psi_{\delta G H}$. In order to decide the existence of the fixed points of $\Psi_{\delta G H}$, we need the following theorem which can be found in [1, page 74].

THEOREM 1.1 (Schauder and Tychonoff). Let $X$ be a compact convex set in a locally convex linear topological space. Then each continuous map $\Psi: X \rightarrow X$ has a fixed point. 
Define a metric $\rho_{m}$ on $C^{m}(\mathbb{R}, \mathbb{R})$, for any $f, g \in C^{m}(\mathbb{R}, \mathbb{R})$, by

$$
\rho_{m}(f, g)=\sup \left\{\left|f^{(j)}(x)-g^{(j)}(x)\right| /\left(1+x^{2}\right): j \in \mathbb{Z}_{m}, x \in \mathbb{R}\right\}
$$

Denote by $0^{*}$ the function on $\mathbb{R}$ which is identical to 0 . For $f \in C^{m}(\mathbb{R}, \mathbb{R})$, write $\|f\|_{m}=\rho_{m}\left(f, 0^{*}\right)$. Then $0 \leq\|f\|_{m} \leq \infty$.

Now we define a metric $\rho_{m} \times \rho_{m}$ on $C^{m}(\mathbb{R}, \mathbb{R}) \times C^{m}(\mathbb{R}, \mathbb{R})$ by

$$
\rho_{m} \times \rho_{m}\left(\left(f_{1}, g_{1}\right),\left(f_{2}, g_{2}\right)\right)=\sqrt{\left[\rho_{m}\left(f_{1}, f_{2}\right)\right]^{2}+\left[\rho_{m}\left(g_{1}, g_{2}\right)\right]^{2}}
$$

for all $\left(f_{1}, g_{1}\right),\left(f_{2}, g_{2}\right) \in C^{m}(\mathbb{R}, \mathbb{R}) \times C^{m}(\mathbb{R}, \mathbb{R})$.

Analogous to the proof of [3, Proposition 3.2], we can obtain the following lemma.

LEMMA 1.2. Suppose that $m \geq 0, K_{0}, \ldots, K_{m}$ are all compact intervals, and $r \geq 0$ is a real number. Let $X_{r}=C^{m}\left(\mathbb{R}, \mathbb{R} ; r, K_{0}, \ldots, K_{m}\right)$ as defined in (1.6). Then $\Psi_{\delta G H} \mid\left(X_{r} \times X_{r}, \rho_{m} \times \rho_{m}\right)$ is continuous.

2. $C^{0}$ solutions of (1.1). For any $G \in C^{0}\left(\mathbb{R}^{2 n+3}, \mathbb{R}\right)$, define a function $\varphi_{G}$ : $\mathbb{R} \rightarrow \mathbb{R}$ by

$$
\varphi_{G}(x)=G(x, x, \ldots, x) \quad \forall x \in \mathbb{R} .
$$

THEOREM 2.1. Let $G, H \in C^{0}\left(\mathbb{R}^{2 n+3}\right.$, $\left.\mathbb{R}\right)$. If the following two conditions hold:

(i) there exist nonnegative real numbers $\mu_{0}, \varepsilon_{0}, c_{1}, \ldots, c_{2 n+1}$ and $b \geq \mu_{0}+\varepsilon_{0}$, $c_{0} \geq \mu>0$ such that

$$
G, H \in C^{0}\left(\mathbb{R}^{2 n+3}, \mathbb{R} ;\left[\mu_{0}, b-\varepsilon_{0}\right],\left[-c_{0},-\mu\right],\left[-c_{1}, c_{1}\right], \ldots,\left[-c_{2 n+1}, c_{2 n+1}\right]\right)
$$

(ii) $\mu_{0} \geq \sum_{i=1}^{2 n+1} c_{i}(b / \mu), \varepsilon_{0} \geq \sum_{i=1}^{2 n+1} c_{i}(b / \mu)$, and $\mu>2 \sum_{i=1}^{2 n+1} c_{i}$, then (1.1) has a solution $\left(f_{0}, g_{0}\right) \in C^{0}(\mathbb{R}, \mathbb{R} ; r,[0, b / \mu]) \times C^{0}(\mathbb{R}, \mathbb{R} ; r,[0, b / \mu])$, where

$$
r=\frac{\left|\varphi_{G}(0)\right|+\sum_{i=1}^{n} i\left(c_{i}+c_{n+1+i}\right)(b / \mu)}{\mu-\sum_{i=1}^{2 n+1} c_{i}} .
$$

PROOF. We arbitrarily choose a constant $\delta \in\left(0,1 / c_{0}\right]$. It follows from condition (i) that $\delta \leq 1 / c_{0} \leq 1 / \mu$. Let $\Psi_{\delta G H}$ be defined as in (1.15). Let $X_{r}=$ $C^{0}(\mathbb{R}, \mathbb{R} ; r,[0, b / \mu])$. Consider any $(f, g) \in X_{r} \times X_{r}$. Write $\bar{f}=\Psi_{\delta G}(f, g)$. If 
$G \in C^{1}\left(\mathbb{R}^{2 n+3}, \mathbb{R}\right)$ and $f, g \in C^{1}(\mathbb{R}, \mathbb{R})$, then for any $x \in \mathbb{R}$, we have $f^{\prime}(x) \in$ $\bar{\Lambda}_{f} \subset[0, b / \mu], g^{\prime}(x) \in \bar{\Lambda}_{g} \subset[0, b / \mu]$, and

$$
\begin{aligned}
\bar{f}^{\prime}(x)= & f^{\prime}(x)+\delta G_{0}^{\prime}\left(V_{f g}(x)\right)+\delta \sum_{i=1}^{n+1} G_{i}^{\prime}\left(V_{f g}(x)\right) \cdot f^{\prime}(x+i-1) \\
& +\delta \sum_{i=2}^{n+2} G_{n+i}^{\prime}\left(V_{f g}(x)\right) \cdot g^{\prime}(x+i-2) .
\end{aligned}
$$

Noting the upper and lower bounds of $G_{i}^{\prime}\left(V_{f g}(x)\right)$ given in condition (i), from (2.4) and condition (ii) we get

$$
\begin{aligned}
\bar{f}^{\prime}(x) & \geq f^{\prime}(x)+\delta \mu_{0}-\delta c_{0} f^{\prime}(x)-\delta \sum_{i=1}^{2 n+1} \frac{c_{i} b}{\mu} \\
& =\left(1-\delta c_{0}\right) f^{\prime}(x)+\delta\left(\mu_{0}-\sum_{i=1}^{2 n+1} \frac{c_{i} b}{\mu}\right) \geq 0, \\
\bar{f}^{\prime}(x) & \leq \delta\left(b-\varepsilon_{0}\right)+\left(1-\delta \mu+\delta \sum_{i=1}^{2 n+1} c_{i}\right) \frac{b}{\mu} \\
& \leq \delta\left(b-\varepsilon_{0}\right)+\frac{(1-\delta \mu) b}{\mu}+\delta \varepsilon_{0}=\frac{b}{\mu} .
\end{aligned}
$$

Combining (2.5) and (2.6), we obtain $\Lambda(\bar{f}) \subset[0, b / \mu]$, that is,

$$
\Lambda\left(\Psi_{\delta G}(f, g)\right) \subset\left[0, \frac{b}{\mu}\right]
$$

If $G \notin C^{1}\left(\mathbb{R}^{2 n+3}, \mathbb{R}\right), f \notin C^{1}(\mathbb{R}, \mathbb{R})$, or $g \notin C^{1}(\mathbb{R}, \mathbb{R})$, then for any two given points $u>v$ in $\mathbb{R}$, we can take $G_{1} \in C^{1}\left(\mathbb{R}^{2 n+3}, \mathbb{R} ;\left[\mu_{0}, b-\varepsilon_{0}\right],\left[-c_{0},-\mu\right]\right.$, $\left.\left[-c_{1}, c_{1}\right], \ldots,\left[-c_{2 n+1}, c_{2 n+1}\right]\right)$ and $f_{1}, g_{1} \in C^{1}(\mathbb{R}, \mathbb{R} ; r,[0, b / \mu])$ such that for all $j \in\{0,1, \ldots, n\}$ and $w \in\{u, v\}$,

$$
\begin{gathered}
f_{1}(w+j)=f(w+j), \quad g_{1}(w+j)=g(w+j), \\
G_{1}\left(V_{f g}(w)\right)=G\left(V_{f g}(w)\right) .
\end{gathered}
$$

Write ${\overline{f_{1}}}^{\prime}=\Psi_{\delta G}\left(f_{1}, g_{1}\right)$. Then by (2.7), we have $\Lambda\left(\overline{f_{1}}\right) \subset[0, b / \mu]$; hence $(\bar{f}(u)-$ $\bar{f}(v)) /(u-v)=\left(\overline{f_{1}}(u)-\overline{f_{1}}(v)\right) /(u-v) \in[0, b / \mu]$. Thus, (2.7) is still valid when $G \notin C^{1}\left(\mathbb{R}^{2 n+3}, \mathbb{R}\right), f \notin C^{1}(\mathbb{R}, \mathbb{R})$, and $g \notin C^{1}(\mathbb{R}, \mathbb{R})$. Therefore, we have

$$
\Psi_{\delta G}\left(C^{0}\left(\mathbb{R}, \mathbb{R} ;\left[0, \frac{b}{\mu}\right]\right) \times C^{0}\left(\mathbb{R}, \mathbb{R} ;\left[0, \frac{b}{\mu}\right]\right)\right) \subset C^{0}\left(\mathbb{R}, \mathbb{R} ;\left[0, \frac{b}{\mu}\right]\right) .
$$


Since $f, g \in C^{0}(\mathbb{R}, \mathbb{R} ;[0, b / \mu])$ are increasing, we have $f(k) \leq f(0)+k(b / \mu)$ and $g(k) \leq g(0)+k(b / \mu)$, for $k=1,2, \ldots, n$. By condition (ii), we get

$$
\begin{aligned}
|\bar{f}(0)|= & \left|f(0)+\delta\left[G\left(V_{f g}(0)\right)-\varphi_{G}(0)\right]+\delta \varphi_{G}(0)\right| \\
\leq & \left(1-\delta \mu+\delta \sum_{i=1}^{n} c_{i}\right)|f(0)|+\delta \sum_{i=1}^{n+1} c_{n+i}|g(0)| \\
& +\delta\left[\left|\varphi_{G}(0)\right|+\sum_{i=1}^{n} i\left(c_{i}+c_{n+1+i}\right)\left(\frac{b}{\mu}\right)\right] \\
\leq & r+\delta\left[\left|\varphi_{G}(0)\right|+\sum_{i=1}^{n} i\left(c_{i}+c_{n+1+i}\right)\left(\frac{b}{\mu}\right)-\left(\mu-\sum_{i=1}^{2 n+1} c_{i}\right) r\right] \\
= & r .
\end{aligned}
$$

By (2.9) and (2.10), we obtain

$$
\Psi_{\delta G}\left(X_{r} \times X_{r}\right) \subset X_{r} .
$$

Similarly, we can obtain that

$$
\Psi_{\delta H}\left(X_{r} \times X_{r}\right) \subset X_{r}
$$

Therefore, it follows from (2.11) and (2.12) that

$$
\Psi_{\delta G H}\left(X_{r} \times X_{r}\right) \subset X_{r} \times X_{r}
$$

By [3, Proposition 3.1], under the metric $\rho_{0} \times \rho_{0}, X_{r} \times X_{r}$ is compact. By Lemma 1.2, $\Psi_{\delta G H} \mid\left(X_{r} \times X_{r}, \rho_{0} \times \rho_{0}\right)$ is continuous. Since $X_{r} \times X_{r}$ is a convex subspace of $C^{0}(\mathbb{R}, \mathbb{R}) \times C^{0}(\mathbb{R}, \mathbb{R})$, by Theorem 1.1, $\Psi_{\delta G H} \mid\left(X_{r} \times X_{r}\right)$ has a fixed point. This implies that (1.1) has a solution $\left(f_{0}, g_{0}\right) \in X_{r} \times X_{r}$. Theorem 2.1 is proven.

For any $h \in C^{0}(\mathbb{R}, \mathbb{R})$, write $B C^{0}(\mathbb{R}, \mathbb{R} ; h)=\left\{f \in C^{0}(\mathbb{R}, \mathbb{R}):\|f-h\|_{0}<\infty\right\}$.

THeOREM 2.2. Suppose that $G, H \in C^{0}\left(\mathbb{R}^{2 n+3}, \mathbb{R}\right)$ satisfy Theorem 2.1(i) and (ii), and $\left(f_{0}, g_{0}\right) \in C^{0}(\mathbb{R}, \mathbb{R} ;[0, b / \mu]) \times C^{0}(\mathbb{R}, \mathbb{R} ;[0, b / \mu])$ is a solution of (1.1). Then (1.1) has only a solution $\left(f_{0}, g_{0}\right)$ in $B C^{0}\left(\mathbb{R}, \mathbb{R} ; f_{0}\right) \times B C^{0}\left(\mathbb{R}, \mathbb{R} ; g_{0}\right)$.

Proof. Suppose that $\left(f_{1}, g_{1}\right) \in B C^{0}\left(\mathbb{R}, \mathbb{R} ; f_{0}\right) \times B C^{0}\left(\mathbb{R}, \mathbb{R} ; g_{0}\right)$ is also a solution of (1.1). Consider the following two cases. 
CASE $1\left(\left\|g_{0}-g_{1}\right\|_{0} \leq\left\|f_{0}-f_{1}\right\|_{0}\right)$. For any $x \in \mathbb{R}$, there exists $w_{i}=w_{i}(x) \in$ $\Lambda_{i G}(i=1, \ldots, 2 n+2)$ such that

$$
\begin{aligned}
0= & G\left(V_{f_{0} g_{0}}(x)\right)-G\left(V_{f_{1} g_{1}}(x)\right) \\
= & w_{1}\left(f_{0}(x)-f_{1}(x)\right)+\sum_{i=2}^{n+1} w_{i}\left(f_{0}(x+i-1)-f_{1}(x+i-1)\right) \\
& +\sum_{i=2}^{n+2} w_{n+i}\left(g_{0}(x+i-2)-g_{1}(x+i-2)\right) \\
\geq & \left|w_{1}\left(f_{0}(x)-f_{1}(x)\right)\right|-\sum_{i=2}^{n+1}\left|w_{i}\left(f_{0}(x+i-1)-f_{1}(x+i-1)\right)\right| \\
& -\sum_{i=2}^{n+2}\left|w_{n+i}\left(g_{0}(x+i-2)-g_{1}(x+i-2)\right)\right| \\
\geq & \mu\left|f_{0}(x)-f_{1}(x)\right|-\sum_{i=1}^{n} c_{i}|| f_{0}-f_{1}\left\|_{0}-\sum_{i=1}^{n+1} c_{n+i}|| g_{0}-g_{1}\right\|_{0} \\
\geq & \mu\left|f_{0}(x)-f_{1}(x)\right|-\sum_{i=1}^{2 n+1} c_{i}|| f_{0}-f_{1} \|_{0} .
\end{aligned}
$$

By (2.14), we have $\left(\mu-\sum_{i=1}^{2 n+1} c_{i}\right)\left\|f_{0}-f_{1}\right\|_{0} \leq 0$. Since $\mu>2 \sum_{i=1}^{2 n+1} c_{i}$, $\| f_{0}-$ $f_{1} \|_{0}=0$ which implies $f_{1}=f_{0}$. It follows from $\left\|g_{0}-g_{1}\right\|_{0} \leq\left\|f_{0}-f_{1}\right\|_{0}=0$ that $g_{1}=g_{0}$. Hence, $\left(f_{1}, g_{1}\right)=\left(f_{0}, g_{0}\right)$.

CASE $2\left(\left\|g_{0}-g_{1}\right\|_{0}>\left\|f_{0}-f_{1}\right\|_{0}\right)$. Analogous to Case 1 , we can also show that $\left(f_{1}, g_{1}\right)=\left(f_{0}, g_{0}\right)$. Thus, $(1.1)$ has only a solution $\left(f_{0}, g_{0}\right)$ in $B C^{0}\left(\mathbb{R}, \mathbb{R} ; f_{0}\right) \times$ $B C^{0}\left(\mathbb{R}, \mathbb{R} ; g_{0}\right)$. Theorem 2.2 is proven.

\section{3. $C^{m}$ solutions $(m \geq 1)$ of $\mathbf{( 1 . 1 )}$}

THEOREM 3.1. Suppose that $G, H \in L C^{m}\left(\mathbb{R}^{2 n+3}, \mathbb{R}\right)$ satisfy Theorem 2.1(i) and (ii). Then there exist positive numbers $a_{1}, \ldots, a_{m}$ such that (1.1) has a solution

$$
\begin{aligned}
\left(f_{m}, g_{m}\right) \in & C^{m}\left(\mathbb{R}, \mathbb{R} ; r,\left[0, \frac{b}{\mu}\right],\left[-a_{1}, a_{1}\right], \ldots,\left[-a_{m}, a_{m}\right]\right) \\
& \times C^{m}\left(\mathbb{R}, \mathbb{R} ; r,\left[0, \frac{b}{\mu}\right],\left[-a_{1}, a_{1}\right], \ldots,\left[-a_{m}, a_{m}\right]\right) .
\end{aligned}
$$

Proof. For any $\delta \in\left(0,1 / c_{0}\right]$, let the map $\Psi_{\delta G H}$ be defined as in (1.15). Write $X_{r m}=C^{m}(\mathbb{R}, \mathbb{R} ; r,[0, b / \mu])$. By (2.13), we have

$$
\Psi_{\delta G H}\left(X_{r m} \times X_{r m}\right) \subset X_{r m} \times X_{r m}
$$


since $G, H \in C^{m}\left(\mathbb{R}^{2 n+3}, \mathbb{R}\right)$. Consider any $f, g \in C^{m}(\mathbb{R}, \mathbb{R} ; r,[0, b / \mu]) \cap L C^{m}(\mathbb{R}$, $\mathbb{R})$. Let $h(x)=G\left(V_{f g}(x)\right)$. Then we can calculate the derivatives of $h$ of order $1,2, \ldots, m$ as follows:

$$
\begin{aligned}
h^{\prime}(x)= & \sum_{i=0}^{n+1} G_{i}^{\prime}\left(V_{f g}(x)\right) \cdot f^{\prime}(x+i-1)+\sum_{i=2}^{n+2} G_{n+i}^{\prime}\left(V_{f g}(x)\right) \cdot g^{\prime}(x+i-2) \\
h^{\prime \prime}(x)= & \sum_{i=1}^{n+1} G_{i}^{\prime}\left(V_{f g}(x)\right) f^{\prime \prime}(x+i-1)+\sum_{i=2}^{n+2} G_{n+i}^{\prime}\left(V_{f g}(x)\right) g^{\prime \prime}(x+i-2) \\
& +\sum_{i=0}^{n+1} \sum_{j=0}^{n+1} G_{i j}^{\prime \prime}\left(V_{f g}(x)\right) f^{\prime}(x+i-1) f^{\prime}(x+j-1) \\
& +\sum_{i=0}^{n+1} \sum_{j=2}^{n+2} G_{i, j+n}^{\prime \prime}\left(V_{f g}(x)\right) f^{\prime}(x+i-1) g^{\prime}(x+j-2) \\
& +\sum_{i=2}^{n+2} \sum_{j=0}^{n+1} G_{n+i, j}^{\prime \prime}\left(V_{f g}(x)\right) f^{\prime}(x+j-1) g^{\prime}(x+i-2) \\
& +\sum_{i=2}^{n+2} \sum_{j=2}^{n+2} G_{n+i, n+j}^{\prime \prime}\left(V_{f g}(x)\right) g^{\prime}(x+i-2) g^{\prime}(x+j-2)
\end{aligned}
$$

where $d x / d x(=1)$ is written as $f^{\prime}(x-1)$ for convenience.

In general, for $k=2, \ldots, m$, it is easy to see that

$$
\begin{aligned}
h^{(k)}(x)= & \sum_{i=1}^{n+1} G_{i}^{\prime}\left(V_{f g}(x)\right) f^{(k)}(x+i-1) \\
& +\sum_{i=2}^{n+2} G_{n+i}^{\prime}\left(V_{f g}(x)\right) g^{(k)}(x+i-2)+\xi_{k}(\{\cdot\},\{\cdot\},\{\cdot\}),
\end{aligned}
$$

where

$$
\begin{aligned}
\xi_{k}(\{\cdot\},\{\cdot\},\{\cdot\})=\xi_{k} & \left\{\left\{G_{i_{1} i_{2} \cdots i_{p}}^{(p)}\left(V_{f g}(x)\right): p \in \mathbb{N}_{k},\left(i_{1}, i_{2}, \ldots, i_{p}\right) \in \mathbb{Z}_{2 n+2}^{p}\right\},\right. \\
& \left\{f^{(p)}(x+q-1): p \in \mathbb{N}_{k-1}, q \in \mathbb{Z}_{n+1}\right\}, \\
& \left.\left\{g^{(p)}(x+q-1): p \in \mathbb{N}_{k-1}, q \in \mathbb{Z}_{n+1}\right\}\right)
\end{aligned}
$$

$\left(d^{p} x / d x^{p}(=1)\right.$ is written as $f^{(p)}(x-1)$ for convenience) is a polynomial of $G_{i_{1} i_{2} \cdots i_{p}}^{(p)}\left(V_{f g}(x)\right.$ ) (where $\left.p \in \mathbb{N}_{k} ; i_{1}, \ldots, i_{p} \in \mathbb{Z}_{2 n+2}\right), f^{(p)}(x+q-1$ ), and $g^{(p)}(x+q-1)$ (where $\left.p \in \mathbb{N}_{k-1}, q \in \mathbb{Z}_{n+1}\right)$ whose coefficients are all positive integers. The functional relation $\xi_{k}$ itself is related only to the rules of partial derivatives of general functions of several variables and the rules of derivatives of compositions and products of functions, but not related to specific $G$, $f$, or $g$. Therefore, $\xi_{k}$ is still well defined for $k>m$. If $G \in C^{m+1}\left(\mathbb{R}^{2 n+3}, \mathbb{R}\right)$ and $f, g \in C^{m+1}(\mathbb{R}, \mathbb{R})$, then (3.4) also holds for $k=m+1$. 
For $k=0,1, \ldots, m$, let

$$
b_{k}=\max \left\{\lambda\left(f^{(k)}\right), \lambda\left(g^{(k)}\right)\right\}, \quad B_{k}=\max \left\{\lambda_{G}^{(j)}, \lambda_{H}^{(j)}: j=0,1, \ldots, k\right\} .
$$

It follows from (1.4) and (1.3) that $b_{k}=\max \left\{\left\|f^{(k+1)}\right\|_{0},\left\|g^{(k+1)}\right\|_{0}\right\}, k=0, \ldots$, $m-1$. Since $G, H \in L C^{m}\left(\mathbb{R}^{2 n+3}, \mathbb{R}\right)$, by (1.12), we have $B_{0} \leq B_{1} \leq \cdots \leq B_{m}<\infty$. For $1 \leq p \leq k \leq m$, by (1.8), (1.9), (1.10), and (1.13), we have $\left|G_{i_{1} i_{2} \cdots i_{p}}^{(p)}\left(V_{f g}(x)\right)\right|$ $\leq B_{k-1}$. Now, we choose a $\delta \in\left(0,1 / c_{0}\right]$ such that

$$
\delta<\frac{1}{2\left(\mu-\sum_{i=1}^{2 n+1} c_{i}\right)} .
$$

Write $\bar{\mu}=\mu-\sum_{i=1}^{2 n+1} c_{i}$ and $\bar{f}=\Psi_{\delta G}(f, g)$. Replacing all $G_{i_{1} i_{2} \cdots i_{p}}^{(p)}\left(V_{f g}(x)\right)$, $f^{(p)}(f(x+q-1))$, and $g^{(p)}(f(x+q-1))$ in the polynomial $\xi_{k}(\{\cdot\},\{\cdot\}$, $\{\cdot\})$ by the upper bounds $B_{k-1}$ and $b_{p-1}$ of their absolute values, from (3.4) we get

$$
\begin{aligned}
\left|\bar{f}^{(k)}(x)\right| & \leq\left(1-\delta \mu+\delta \sum_{i=1}^{2 n+1} c_{i}\right) b_{k-1}+\delta \eta_{k}\left(B_{k-1}, b_{0}, b_{1}, \ldots, b_{k-2}\right) \\
& =(1-\delta \bar{\mu}) b_{k-1}+\delta \eta_{k}\left(B_{k-1}, b_{0}, \ldots, b_{k-2}\right), \quad k=2, \ldots, m
\end{aligned}
$$

where $\eta_{k}\left(B_{k-1}, b_{0}, \ldots, b_{k-2}\right)$ is a polynomial of $B_{k-1}, b_{0}, \ldots, b_{k-2}$, whose coefficients are all positive integers. The functional relation $\eta_{k}$ itself is determined by $\xi_{k}$ and is independent of specific $G, H, f$, and $g$. Therefore, $\eta_{k}$ is still well defined for $k>m$.

For $k=2, \ldots, m$, noting that $\lambda\left(\bar{f}^{(k-1)}\right)=\left\|\bar{f}^{(k)}\right\|_{0}$, by (3.8) we obtain

$$
\lambda\left(\bar{f}^{(k-1)}\right) \leq(1-\delta \bar{\mu}) b_{k-1}+\delta \eta_{k}\left(B_{k-1}, b_{0}, \ldots, b_{k-2}\right) .
$$

If $G, H \in C^{m+1}\left(\mathbb{R}^{2 n+3}, \mathbb{R}\right)$ and $f, g \in C^{m+1}(\mathbb{R}, \mathbb{R})$, then $\bar{f} \in C^{m+1}(\mathbb{R}, \mathbb{R})$, (3.8) and (3.9) are also true for $k=m+1$. Adopting the method that is used in the proof of Theorem 2.1 to show that (2.7) still holds when $G \notin C^{1}\left(\mathbb{R}^{2 n+3}, \mathbb{R}\right)$, $f \notin C^{1}(\mathbb{R}, \mathbb{R})$, or $g \notin C^{1}(\mathbb{R}, \mathbb{R})$, we can verify that (3.9) still holds for $k=m+1$ even if $G, H \notin C^{m+1}\left(\mathbb{R}^{2 n+3}, \mathbb{R}\right)$ or $f, g \notin C^{m+1}(\mathbb{R}, \mathbb{R})$.

Let $a_{0}=b / \mu$ and

$$
a_{k-1}=\frac{\eta_{k}\left(B_{k-1}, a_{0}, a_{1}, \ldots, a_{k-2}\right)}{\bar{\mu}}, \quad k=2, \ldots, m+1 .
$$


Then $a_{k-1}$ only depends on $G$ and $H$. Since $\eta_{k}\left(B_{k-1}, b_{0}, b_{1}, \ldots, b_{k-2}\right)$ is a monotone increasing function of $b_{0}, b_{1}, \ldots, b_{k-2}$, and $1-\delta \bar{\mu}>\delta \bar{\mu}>0$, from (3.9) and (3.10) it follows that if $b_{i} \leq a_{i}$ for all $i \in \mathbb{Z}_{k-1}$, then

$$
\lambda\left(\bar{f}^{(k-1)}\right) \leq(1-\delta \bar{\mu}) a_{k-1}+\delta \eta_{k}\left(B_{k-1}, a_{0}, \ldots, a_{k-2}\right)=a_{k-1}, \quad k=2, \ldots, m+1 .
$$

Write $X_{m}=C^{m}\left(\mathbb{R}, \mathbb{R} ; r,[0, b / \mu],\left[-a_{1}, a_{1}\right], \ldots,\left[-a_{m}, a_{m}\right]\right)$. Then $X_{m}$ is a convex subset of $C^{m}(\mathbb{R}, \mathbb{R})$. By (3.2) and (3.11), we get

$$
\Psi_{\delta G}\left(X_{m} \times X_{m}\right) \subset X_{m}
$$

Similarly, we can obtain that

$$
\Psi_{\delta H}\left(X_{m} \times X_{m}\right) \subset X_{m}
$$

Therefore, it follows from (3.12) and (3.13) that

$$
\Psi_{\delta G H}\left(X_{m} \times X_{m}\right) \subset X_{m} \times X_{m}
$$

By [3, Proposition 3.1], under the metric $\rho_{m} \times \rho_{m}, X_{m} \times X_{m}$ is compact. By Lemma 1.2, $\Psi_{\delta G H} \mid\left(X_{m} \times X_{m}, \rho_{m} \times \rho_{m}\right)$ is continuous. Thus, by Theorem 1.1, $\Psi_{\delta G H} \mid\left(X_{m} \times X_{m}\right)$ has a fixed point. This implies that (1.1) has a solution $\left(f_{m}\right.$, $\left.g_{m}\right) \in X_{m} \times X_{m}$. Theorem 3.1 is proven.

Noting that $C^{m}(\mathbb{R}, \mathbb{R}) \subset C^{0}(\mathbb{R}, \mathbb{R})$, we have the following proposition.

Proposition 3.2. Let $G, H \in C^{0}\left(\mathbb{R}^{2 n+3}, \mathbb{R}\right), \mathbf{F} \subset C^{0}(\mathbb{R}, \mathbb{R}) \times C^{0}(\mathbb{R}, \mathbb{R})$, and $m \geq 1$. If (1.1) has at most a solution in $\mathbf{F}$, then (1.1) has at most a solution in $\mathbf{F} \cap\left(C^{m}(\mathbb{R}, \mathbb{R}) \times C^{m}(\mathbb{R}, \mathbb{R})\right)$.

By Proposition 3.2, after obtaining Theorem 2.2, we need not discuss the uniqueness of $C^{m}$ solutions of (1.1), for $m \geq 1$, in detail unless we can give some conditions weaker than those in Theorem 2.2 (or, at least, they do not imply each other) or we can discuss the uniqueness of solutions in a subspace of $C^{0}(\mathbb{R}, \mathbb{R}) \times C^{0}(\mathbb{R}, \mathbb{R})$ larger than the subspace of $B C^{0}\left(\mathbb{R}, \mathbb{R} ; f_{m}\right) \times B C^{0}\left(\mathbb{R}, \mathbb{R} ; g_{m}\right)$.

4. Example. Let $a \geq 10$ be a real number. Suppose that the system of equations is

$$
\begin{gathered}
\frac{3}{2} x-a f(x)+\sin f(x) \cdot \cos f(x+1)+\frac{1}{2} \sin (2 g(x)-1)+\arctan g(x+1)=0, \\
2 x+\sin (x-g(x+1)+f(x)+f(x+1))-\bar{a} g(x)+\arctan g(x)=0,
\end{gathered}
$$


for all $x \in \mathbb{R}$. Then the representatives of the corresponding $G, H: \mathbb{R}^{5} \rightarrow \mathbb{R}$ are

$$
\begin{gathered}
G\left(x_{0}, x_{1}, x_{2}, x_{3}, x_{4}\right)=\frac{3}{2} x_{0}-a x_{1}+\sin x_{1} \cdot \cos x_{2}+\frac{1}{2} \sin \left(2 x_{3}-1\right)+\arctan x_{4}, \\
H\left(x_{0}, x_{1}, x_{2}, x_{3}, x_{4}\right)=2 x_{0}+\sin \left(x_{0}-x_{2}+x_{3}+x_{4}\right)-\bar{a} x_{1}+\arctan x_{1},
\end{gathered}
$$

for all $\left(x_{0}, x_{1}, x_{2}, x_{3}, x_{4}\right) \in \mathbb{R}^{5}$. We can easily calculate the derivatives of $G$ and $H$, and then obtain the supremums and infimums of $\Lambda_{i G}$ and $\Lambda_{i H}(i=$ $0,1,2,3,4)$; hence we can take $\mu_{0}=1, b=3+\varepsilon_{0}\left(\varepsilon_{0} \geq 0\right.$ is to be selected), $c_{0}=a+1, \mu=a-1$, and $c_{1}=c_{2}=c_{3}=1$.

When $a+1>\bar{a} \geq a \geq 13$, we choose $\varepsilon_{0}=(a-10) / 3$. Then $b / \mu=1 / 3$ and Theorem 2.1(i) and (ii) hold. Thus, by Theorems 2.1, 2.2, 3.1, and Proposition 3.2 , we have the following proposition.

Proposition 4.1. Let $m \geq 0$ be given. If $a+1>\bar{a} \geq a \geq 13$, then (4.1) has $a$ solution $\left(f_{m}, g_{m}\right) \in C^{m}(\mathbb{R}, \mathbb{R} ;[0,1 / 3]) \times C^{m}(\mathbb{R}, \mathbb{R} ;[0,1 / 3])$, and $\left(f_{m}, g_{m}\right)$ is the unique solution of $(4.1)$ in $B C^{0}\left(\mathbb{R}, \mathbb{R} ; f_{m}\right) \times B C^{0}\left(\mathbb{R}, \mathbb{R} ; g_{m}\right)$.

Consider the following system of equations:

$$
\begin{aligned}
x+\arctan x- & 19 f(x)+e^{-\left[(f(x+2))^{2}+(f(x+5))^{2}+(g(x))^{2}+(g(x+8))^{2}\right]}=0, \\
\frac{3}{2} x+1- & 20 g(x)+\ln \left[(f(x+3))^{2}+(g(x+4))^{2}+1\right] \\
+ & \sin (g(x+5)) \cdot \cos (f(x)+g(x+2))=0 .
\end{aligned}
$$

Analogous to the argument of Proposition 4.1, we can obtain the following proposition.

Proposition 4.2. Let $m \geq 0$ be given. Equation (4.3) has a solution ( $f_{m}$, $\left.g_{m}\right) \in C^{m}(\mathbb{R}, \mathbb{R} ;[0,1 / 6]) \times C^{m}(\mathbb{R}, \mathbb{R} ;[0,1 / 6])$, and it is the unique solution of (4.3) in $B C^{0}\left(\mathbb{R}, \mathbb{R} ; f_{m}\right) \times B C^{0}\left(\mathbb{R}, \mathbb{R} ; g_{m}\right)$.

AcKnowledgments. The authors would like to express their sincere appreciation to the referees for their very helpful suggestions and many kind comments. This work was supported by the National Natural Science Foundation of China Grant 10226014 and the Guangxi Science Foundation Grant 0229001.

\section{REFERENCES}

[1] J. Dugundji and A. Granas, Fixed Point Theory. I, Monografie Matematyczne, vol. 61, Polish Scientific Publishers, Warsaw, 1982.

[2] X. Liu, J. Ma, and X. Zhao, $C^{m}$ solutions of nonhomogeneous linear difference equations, Math. Appl. 15 (2002), no. 3, 144-148.

[3] J. Mai and X. Liu, Existence, uniqueness and stability of $\mathrm{C}^{m}$ solutions of iterative functional equations, Sci. China Ser. A 43 (2000), no. 9, 897-913.

[4] A. Mukherjea and J. S. Ratti, On a functional equation involving iterates of a bijection on the unit interval, Nonlinear Anal. 7 (1983), no. 8, 899-908. 
[5] _ A functional equation involving iterates of a bijection on the unit interval. II, Nonlinear Anal. 31 (1998), no. 3-4, 459-464.

[6] R. E. Rice, B. Schweizer, and A. Sklar, When is $f(f(z))=a z^{2}+b z+c$ ? Amer. Math. Monthly 87 (1980), no. 4, 252-263.

[7] W. Zhang, Discussion on the iterated equation $\sum_{i=1}^{n} \lambda_{i} f^{i}(x)=F(x)$, Chinese Sci. Bull. 32 (1987), no. 21, 1444-1451.

[8] _ Discussion on the differentiable solutions of the iterated equation $\sum_{i=1}^{n} \lambda_{i} f^{i}(x)=F(x)$, Nonlinear Anal. 15 (1990), no. 4, 387-398.

Xinhe Liu: Department of Mathematics, Guangxi University, Nanning 530004, China E-mail address: xh1wh1@gxu.edu.cn

Xiuli Zhao: Department of Mathematics, Guangxi University, Nanning 530004, China Jianmin Ma: Department of Mathematics, Guangxi University, Nanning 530004, China 


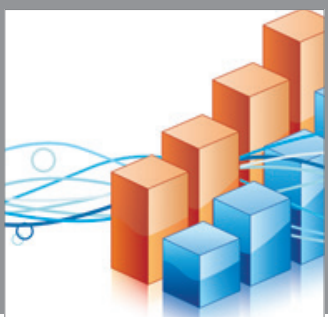

Advances in

Operations Research

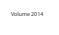

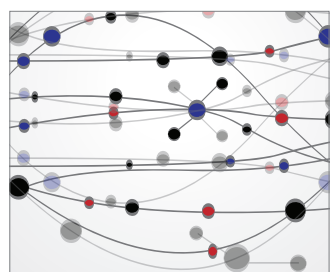

\section{The Scientific} World Journal
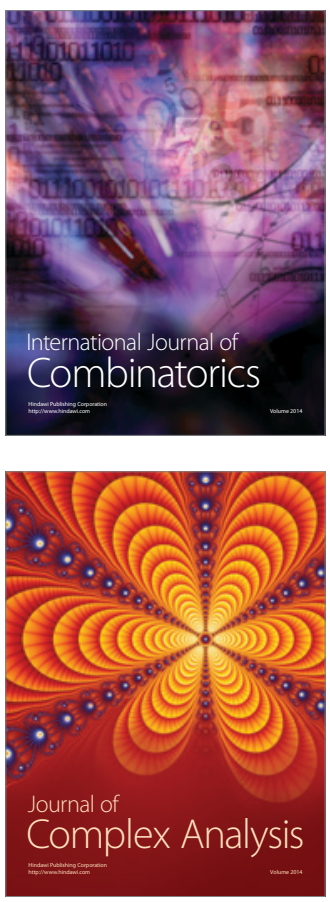

International Journal of

Mathematics and

Mathematical

Sciences
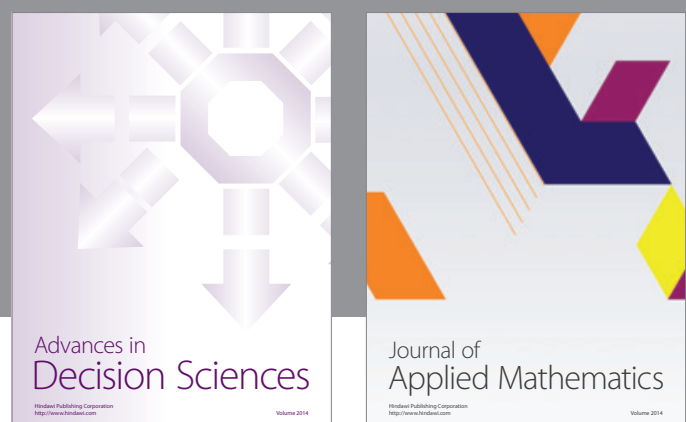

Journal of

Applied Mathematics
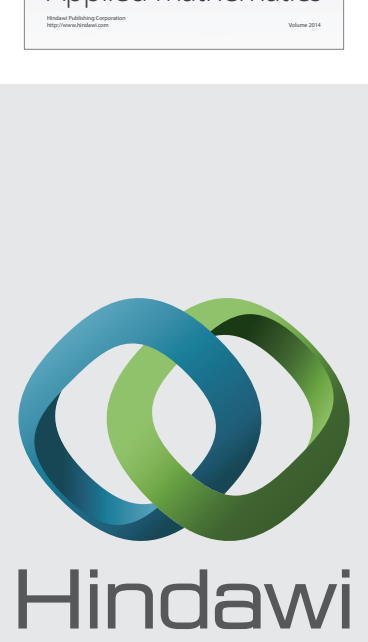

Submit your manuscripts at http://www.hindawi.com
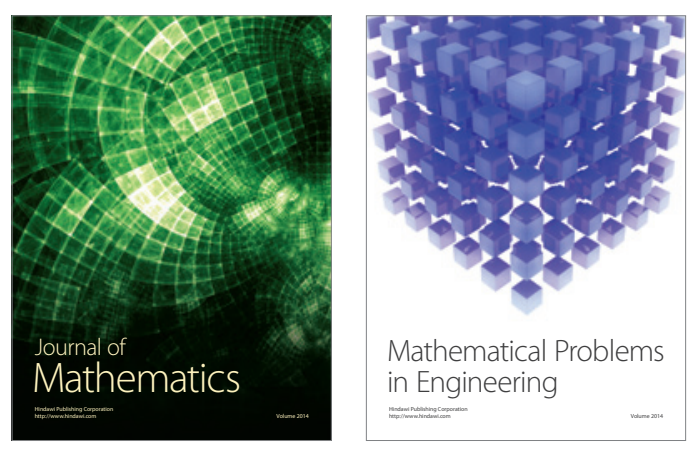

Mathematical Problems in Engineering
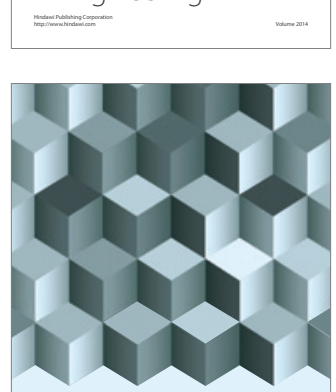

Journal of

Function Spaces
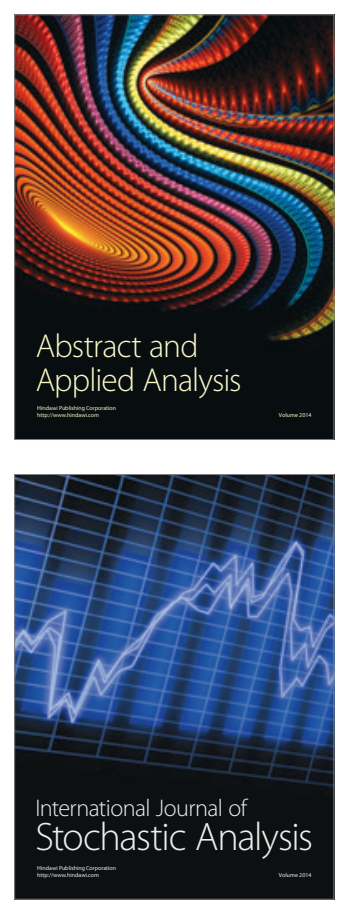

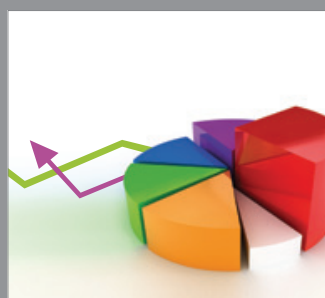

ournal of

Probability and Statistics

Promensencen
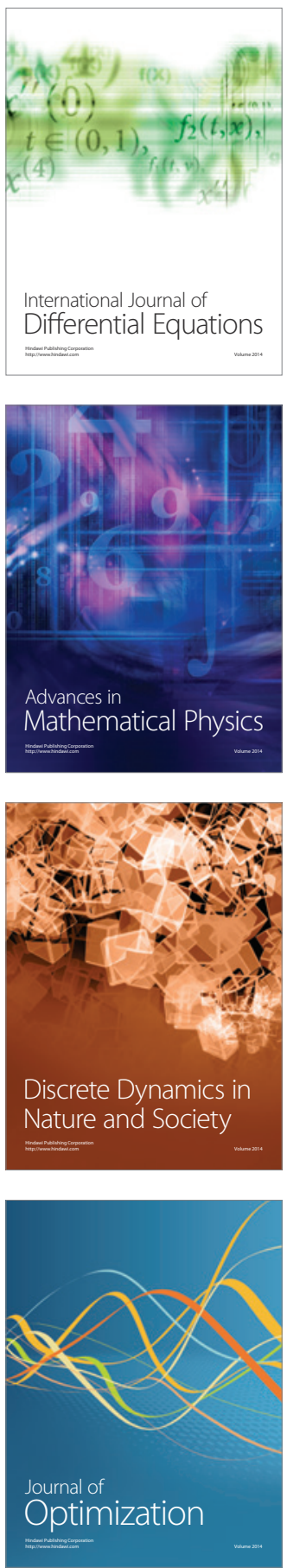